\title{
Investigation of morphological changes in platinum-containing nanostructures created by electron-beam-induced deposition
}

\author{
A. Botman ${ }^{\text {a) }}$ \\ Faculty of Applied Sciences, Delft University of Technology, Lorentzweg 1, 2628 CJ Delft, The Netherlands \\ M. Hesselberth \\ Kamerlingh Onnes Laboratory, Leiden Institute of Physics, Universiteit Leiden, 2300 RA Leiden, \\ The Netherlands \\ J. J. L. Mulders \\ FEI Electron Optics, Achtseweg Noord 5, 5600 KA Eindhoven, The Netherlands
}

(Received 18 June 2008; accepted 2 September 2008; published 1 December 2008)

\begin{abstract}
Focused electron-beam-induced deposition (EBID) allows the rapid fabrication of three-dimensional nanodevices and metallic wiring of nanostructures, and is a promising technique for many applications in nanoresearch. The authors present two topics on platinum-containing nanostructures created by EBID. First, they report on a TEM study of the microstructure of nanodeposits created from $\mathrm{Pt}\left(\mathrm{PF}_{3}\right)_{4}$. They have performed imaging and electron energy loss spectroscopy with a transmission electron microscope (TEM). The deposited material, composed mainly of platinum, phosphor, oxygen, and trace amounts of fluorine, is amorphous. Platinum is evenly distributed in the nanostructure, while phosphorus is observed to cluster. The size and amount of phosphorus clusters depend on the thickness of the structure. Second, they document an aging process in structures created from $\mathrm{MeCpPtMe}_{3}$, which have a resistivity which increases with the time they are exposed to air, from $2 \times 10^{5}$ to $1.8 \times 10^{8} \mu \Omega \mathrm{cm}$ over 55 days. They demonstrate that covering the structures with an EBID-deposited insulating protective layer of TEOS can prevent this oxidation. In doing so, they achieved the lowest reported resistivity for EBID from this platinum precursor, of 1.5 $\times 10^{3} \mu \Omega \mathrm{cm}$. @ 2008 American Vacuum Society. [DOI: 10.1116/1.2990790]
\end{abstract}

\section{INTRODUCTION}

Electron-beam-induced deposition (EBID) (Refs. 1 and 2) is a promising technique for nanoscale rapid prototyping and nanolithography alternative. ${ }^{3}$ It is a direct-write process capable of creating conductive or insulating three-dimensional nanostructures within a few minutes inside a scanning electron microscope (SEM); the deposited material depends on the precursor chosen. It can be likened to a local chemical vapor deposition process, with the decomposition of the gaseous precursor caused by the interaction of an electron beam with a solid substrate. The energy transferred from the electron beam to the adsorbed precursor gas molecules in the vicinity of the beam irradiation point dissociates the precursor into a volatile and nonvolatile part. The nonvolatile part collects at the substrate and forms local material growth. Typical applications of EBID include nanowire deposition, ${ }^{4}$ contacting carbon nanotubes, ${ }^{5}$ and growing tips for field emission $^{6}$ and atomic and magnetic force microscopy. ${ }^{7}$

\section{AGING IN STRUCTURES CREATED FROM $\mathrm{MeCpPtMe}_{3}$}

\section{A. Introduction and method}

One application for EBID is to deposit conductive lines at a position chosen by the SEM operator, for instance, to pro-

a) Author to whom correspondence should be addressed; electronic mail: a.p.j.m.botman@tudelft.nl vide contacts for measuring the transport properties of carbon nanotubes. Therefore it is often desired that the deposited material's resistivity be as close as possible to the bulk metal resistivity, namely, $10.62 \mu \Omega \mathrm{cm}$. Usually however, for the standard platinum precursor methylcyclopentadienyl platinum trimethyl $\left(\mathrm{MeCpPtMe}_{3}\right)$, values reported in literature are greater than $1 \times 10^{5} \mu \Omega \mathrm{cm}$ and differ greatly from one publication to the other. Part of the variability in the reported values are due to different deposition conditions, which can affect the material properties significantly ${ }^{8}$ or varying degrees of carbon contamination within the chamber. Unfortunately the exact deposition conditions are not always reported. Here we document an additional source of variability, namely, the amount of time between the deposition event and the measurement event. For this specific precursor deposited in the specific way described below, the resistivity of the resulting structure is dependent on this time.

An FEI Nova NanoSEM was used at $20 \mathrm{kV}$ and $2.4 \mathrm{nA}$ electron beam current to deposit lines with size of 8.5 $\times 0.8 \mu \mathrm{m}^{2}$ for $6 \mathrm{~min}$ from methylcyclopentadienyl platinum trimethyl ( $\mathrm{MeCpPtMe}_{3}, \mathrm{CAS}$ : 94442-22-5), with a dwell time of $2 \mu \mathrm{s}$. The structures were deposited across fourpoint-probe structures, as shown in Fig. 1 such that the conductivity could be measured. After deposition and between measurements, the samples were stored in air for various lengths of time.

Resistivity measurements were performed in a four-pointprobe station, with a Keithley current source supplying $1 \mathrm{nA}$ 


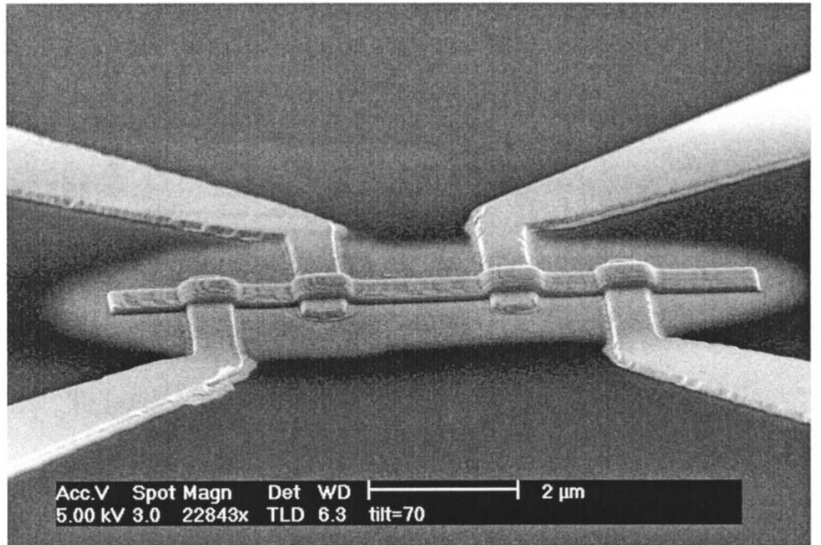

FIG. 1. SEM image (at $70^{\circ}$ tilt) of a typical EBID-deposited structure across four gold electrodes on $\mathrm{Si}_{3} \mathrm{~N}_{4}$ for four-point-probe resistivity characterization. The halo does not contribute to the measurement in a significant manner.

in series with a $1 \mathrm{G} \Omega$ resistance, and a Keithley nanovoltmeter. The voltmeter readout is then linear with the resistivity which can be obtained if the exact geometry of the deposited structure is known.

\section{B. Results and Discussion}

Measurements performed at different time intervals on nine samples are shown in Fig. 2. A measured potential difference of $0.04 \mathrm{mV}$ is equivalent to around $2 \times 10^{5} \mu \Omega \mathrm{cm}$ in the case of our structures, although the exact resistivity values are dependent on the exact height of each of the structures. It is clear that the measured value increases with time, for instance, to $35 \mathrm{mV}\left(1.8 \times 10^{8} \mu \Omega \mathrm{cm}\right)$ after 55 days. Each sample (deposited in the same conditions) appears to age in the same qualitative manner. The different slopes might be attributable to samples of slightly different heights. A curve of power form $V=\gamma+\alpha t^{\beta}$ can be fitted to the data, with $\beta$ values of $0.6 \pm 0.1$.

Further nanostructures were also created in the same way but additionally, immediately after deposition of the platinum

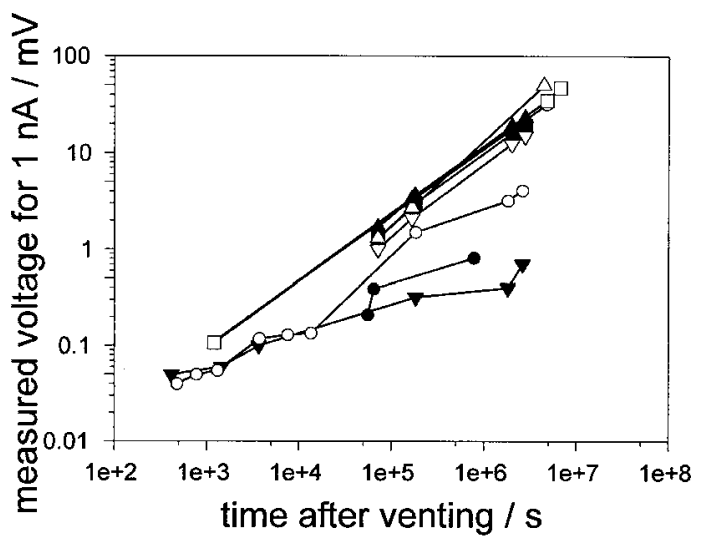

FIG. 2. The measured voltage drop across the EBID-deposited structures for $1 \mathrm{nA}$ (hence a measure of resistivity) is shown as a function of time after the structures are exposed to air.

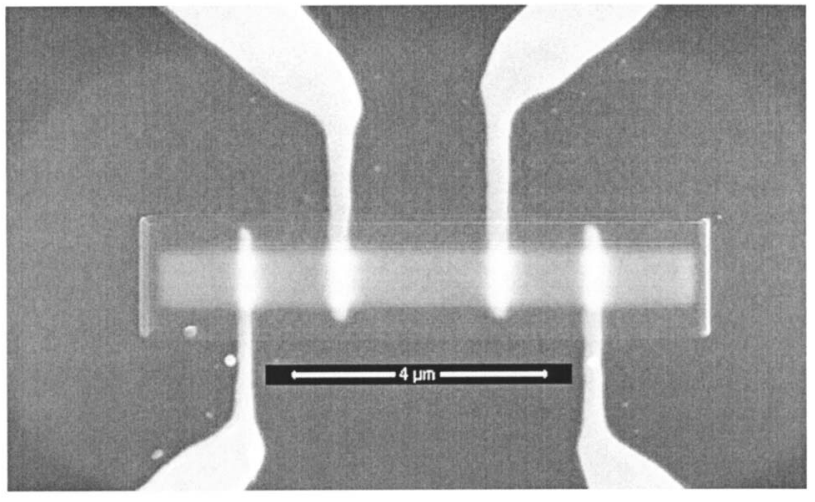

FIG. 3. SEM image of platinum structure across four gold electrodes on $\mathrm{Si}_{3} \mathrm{~N}_{4}$. The platinum deposit is covered with a TEOS deposit to prevent exposure of the platinum structure to air. The TEOS layer is somewhat transparent to electrons at imaging parameters used $(20 \mathrm{kV}$ and $2.4 \mathrm{nA})$.

strip, in the same vacuum chamber, electrically insulating material [tetraethylorthosilicate (TEOS) mixed with a small amount of water vapor] was deposited on top. This was performed in such a manner as to prevent exposure of the platinum strip to air. The deposition parameters for this protective layer were $20 \mathrm{kV}$ beam at $2.4 \mathrm{nA}$, dwell time $200 \mathrm{~ns}$ for $10 \mathrm{~min}$ over $9.5 \times 1.8 \mu \mathrm{m}$. The platinum nanostructures covered with this protective layer were therefore never exposed to air. A SEM image of the resulting structure is shown in Fig. 3. Independent measurements of the protective layer material's resistivity gave a value greater than $10^{14} \mu \Omega \mathrm{cm}$. We verified that the resistivity was indeed higher than could be measured with our setup. The resulting resistivity of the protected strips was much improved, at $1.5 \times 10^{3} \mu \Omega \mathrm{cm}$. We did not observe aging on these structures.

The aging process observed in our uncovered structures might be either chemical or physical in nature. In principle, with such low platinum content the structure presents a percolation network of platinum clusters embedded in an amorphous carbon matrix. There is good evidence for this in literature either in the form of transmission electron microscope (TEM) images or other variants of $I V$ measurements. ${ }^{9}$ Our first idea was that the platinum would migrate and cause cluster growth. This would increase the average distance between clusters, which decreases the conductivity; this would be a physical aging process. However we have evidence that this is not the correct explanation, as follows.

Our data indicate that the "clock" for the aging process starts ticking once the sample is brought out of vacuum and into contact with air. For instance, we deposited two structures with a $1 \mathrm{~h}$ interval between them, then brought the samples to air and measured them both immediately and after $1 \mathrm{~h}$. The measurement after $1 \mathrm{~h}$ indicated the same change for both samples. Therefore the aging process is somehow activated by the presence of air. The prevention of air contact with a protective oxide layer and the subsequent time stability provides additional evidence that contact with air enables the aging. We therefore believe that the conduc- 


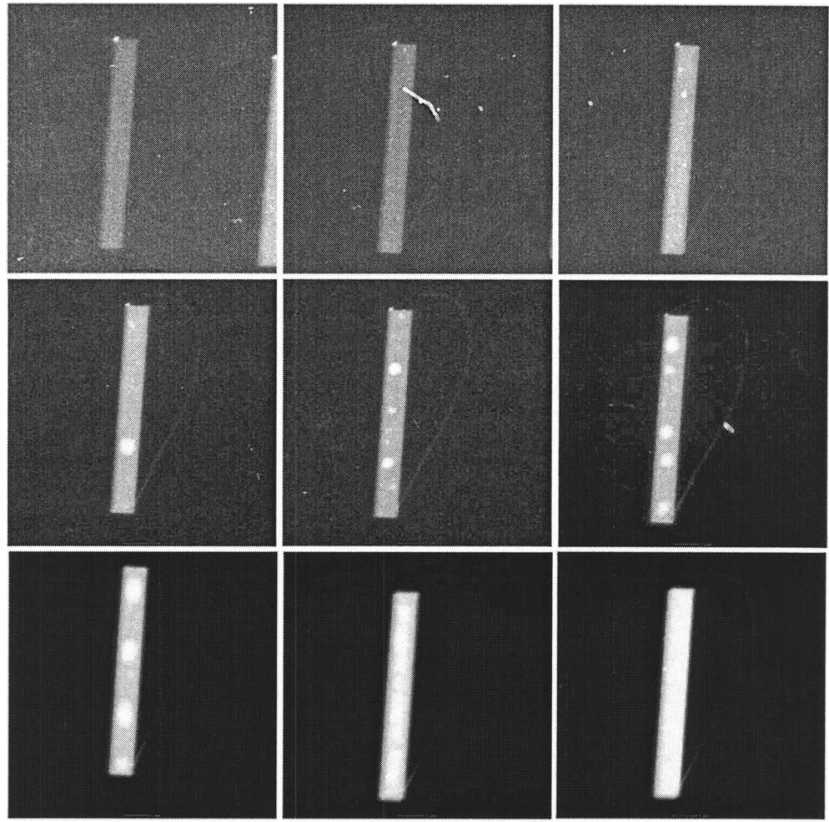

FIG. 4. STEM-HAADF images of nine different deposits, with increasing thickness (deposition time). The presence of whiter areas can be seen, whose number and size increase with deposit thickness.

tivity deterioration is caused by chemical aging, that is, by the oxidation of platinum dispersed in the carbon matrix.

It might be argued that the act of measurement would affect the structures. However confirmation that the behavior presented here is not current induced may be seen in the data itself. Some structures were not measured until quite some time (many days) after deposition. Some structures were measured often while others were measured rarely. In all cases, however, the data are consistent, which indicates that the dominant effect is not measurement induced.

\section{TEM ANALYSIS OF THIN STRUCTURES CREATED FROM Pt $\left(\mathrm{PF}_{3}\right)_{4}$}

\section{A. Introduction and method}

In the case where platinum metal is to be deposited, the precursor $\mathrm{MeCpPtMe}_{3}$ (described in the previous section) is generally used. This precursor, being organometallic, results in carbon-rich material being deposited rather than pure platinum, and the resistivity of the resulting nanostructure is usually high. This low purity and conductivity is often detrimental to the target application. Therefore we have started experiments with a carbon-free precursor, $\mathrm{Pt}\left(\mathrm{PF}_{3}\right)_{4}$ (tetrakis trifluorophosphine platinum, CAS No. 19529-53-4). ${ }^{10}$ The resulting resistivity is better than that of the traditional precursor, at $(690 \pm 30) \mu \Omega \mathrm{cm}$. Here we present a study of the deposited material's microstructure using a TEM. An FEI Nova NanoSEM equipped with a gas injection system was used at $5 \mathrm{kV}$ and $0.9 \mathrm{nA}$ beam current. The pressure in the chamber $3 \times 10^{-5}$ mbar before deposition and $9 \times 10^{-5}$ mbar during deposition, the substrate was a copper TEM grid with thin carbon film $(50 \mathrm{~nm})$. Each structure had

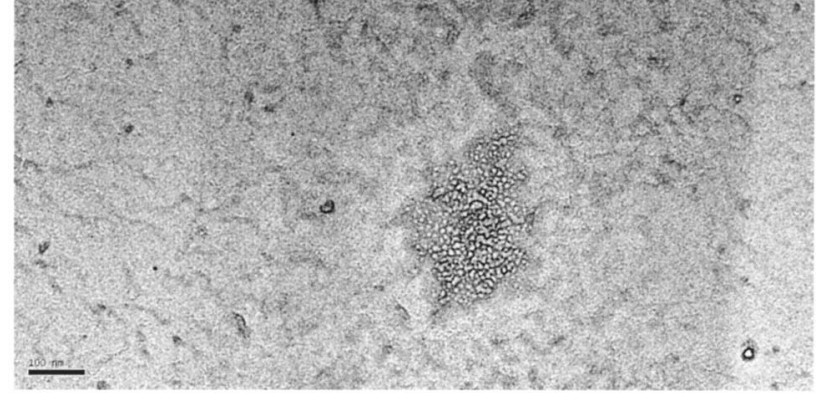

FIG. 5. STEM image of substructure (one cluster). The scale bar is $100 \mathrm{~nm}$.

a footprint of $0.8 \times 8.5 \mu \mathrm{m}$ and was deposited with $1 \mu \mathrm{s}$ dwell time, $0 \%$ overlap. The deposition times ranged from $10 \mathrm{sec}$ to $15 \mathrm{~min}$. After deposition the structures were stored in air.

The structures were analyzed with a FEI Tecnai TEM operating at $200 \mathrm{kV}$. TEM and scanning transmission electron microscope high angle annular dark field (STEM-HAADF) images were taken, and electron dispersive spectroscopy/ electron energy loss spectroscopy (EDX/EELS) mapping was performed.

\section{B. Results and discussion}

From previous experiments we know the bulk composition of the created structures to be (Pt:P:F:C) 48:36:16:0 $( \pm 2)$ at. $\% .^{11}$ The STEM-HAADF images of structures with deposition time 10, 20, $30 \mathrm{~s}$ and 1 (twice), 2, 5, 10, and 15 min are shown sequentially in Fig. 4. The STEM images show areas of increased contrast whose size and number increase with increasing structure thickness. A high resolution TEM image is shown in Fig. 5.

In Fig. 6 we present the result of EDX/EELS mapping on a selected area of the structure. From the results it is clear that the white clusters observed in the STEM images are principally phosphorus. Platinum is uniformly spread across the entire structure. All material observed was amorphous. There was no evidence of grains or crystallinity. We have also measured the resistivity of these structures using the
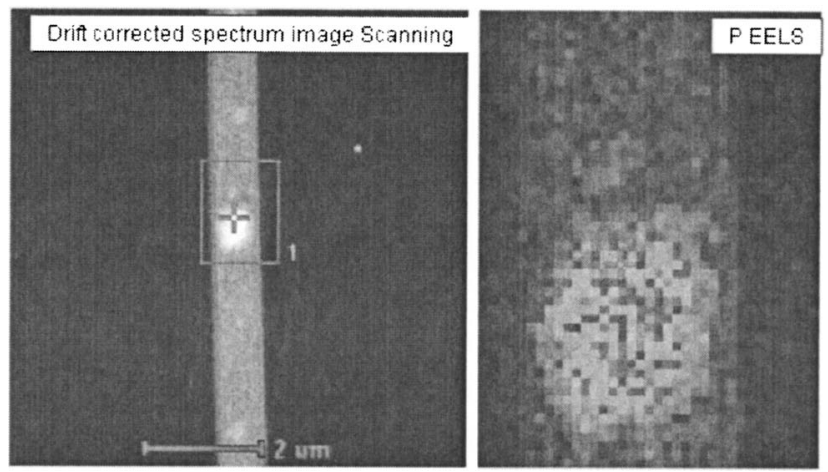

FIG. 6. TEM-EDX/EELS of a selected region of the structure. Left: STEM image. Right: phosphorus EELS. The clusters are phosphorus-rich material. Platinum is distributed uniformly over the entire structure. 
same procedure as in the previous section, over extended periods of time, and we have not seen any aging effects, unlike with the $\mathrm{MeCpPtMe}_{3}$ structures. We suggest this is due to the initially better conductivity $(690 \mu \Omega \mathrm{cm}){ }^{11}$

\section{CONCLUSIONS}

First, we have performed TEM analysis of thin structures created with EBID from $\mathrm{Pt}\left(\mathrm{PF}_{3}\right)_{4}$. We conclude that the results are consistent with bulk EDX values reported to date and that the deposited material is amorphous. We present the surprising fact that during the deposition process, phosphorus appears to cluster. The size and number of these clusters increase with the structure thickness. It is currently not clear why or how this clustering takes place.

Second, we report on the discovery of an aging process in structures created with EBID from $\mathrm{MeCpPtMe}_{3}$. The resistivity of these platinum-containing structures increases after exposure to air, from $2 \times 10^{5}$ to $1.8 \times 10^{8} \mu \Omega \mathrm{cm}$ over 55 days. The evolution appears to follow a power law and seems to be caused by a chemical mechanism (oxidation from exposure to air) rather than a physical mechanism (migration of platinum into clusters). It may be interesting in the future to investigate the temperature dependence of the aging process, which may help to further understand the mechanism behind this phenomenon. Covering the platinum nanostructures with a protective oxide layer using TEOS successfully prevented the aging process and resulted in a resistivity of $1.5 \times 10^{3} \mu \Omega \mathrm{cm}$, which is the lowest resistivity reported to date for EBID from this carbon-based precursor without additional in situ or post treatments. We suggest that authors, when reporting on resistivity values for EBID structures that are not in the metallic regime, give an indication of the time interval between deposition and measurement to enable better comparisons between published results.

\section{ACKNOWLEDGMENTS}

The authors are grateful to Anna Carlsson for her help with the TEM analysis, and thank Pieter Kruit and Kees Hagen for helpful discussions. This research was funded in part by an EU Marie-Curie fellowship, 6th Framework Program, Grant No. FP6-007832-NAPS and in part by FEI Electron Optics.

${ }^{1}$ S. J. Randolph, J. D. Fowlkes, and P. D. Rack, Crit. Rev. Solid State Mater. Sci. 31, 55 (2006).

${ }^{2}$ A. Botman, J. J. L. Mulders, R. Weemaes, and S. Mentink, Nanotechnology 17, 3779 (2006).

${ }^{3}$ W. F. van Dorp, B. van Someren, C. W. Hagen, P. Kruit, and P. A. Crozier, Nano Lett. 5, 1303 (2005).

${ }^{4}$ K. Molhave, D. N. Madsen, S. Dohn, and P. Boggild, Nanotechnology 15, 1047 (2004).

${ }^{5}$ T. Brintlinger, M. S. Fuhrer, J. Melngailis, I. Utke, T. Bret, A. Perentes, P. Hoffmann, M. Abourida, and P. Doppelt, J. Vac. Sci. Technol. B 23, 3174 (2005).

${ }^{6}$ H. W. P. Koops, C. Schoessler, A. Kaya, and M. Weber, J. Vac. Sci. Technol. B 14, 4105 (1996).

${ }^{7}$ Y. M. Lau, P. C. Chee, J. T. L. Thong, and V. Ng, J. Vac. Sci. Technol. A 20, 1295 (2002).

${ }^{8}$ D. Beaulieu, Y. Ding, Z. L. Wang, and W. J. Lackey, J. Vac. Sci. Technol. B 23, 2151 (2005)

${ }^{9}$ L. Rotkina, S. Oh, J. N. Eckstein, and S. V. Rotkin, Phys. Rev. B 72, 233407 (2005)

${ }^{10}$ J. D. Barry, M. Ervin, J. Molstad, A. Wickenden, T. Brintlinger, P. Hoffman, and J. Melngailis, J. Vac. Sci. Technol. B 24, 3165 (2006).

${ }^{11}$ A. Botman, M. Hesselberth, and J. J. L. Mulders, Microelectron. Eng. 85, 1139 (2008). 